\title{
Estimativa de aborto induzido: comparação entre duas metodologias
}

\author{
Maria Teresa Anselmo Olinto e Djalma de Carvalho Moreira Filho²
}

Como citar

Olinto MTA, Moreira Filho D de C. Estimativa de aborto induzido: comparação entre duas metodologias. Rev Panam Salud Publica. 2004;15(5):331-6.

RESUMO Objetivo. Comparar duas metodologias, o método da urna e o método das questões indiretas, para estimar a freqüência de aborto induzido em estudos de base populacional.

Métodos. Foi realizado um estudo transversal de base populacional com uma amostra representativa de 3002 mulheres de 15 a 49 anos residentes na zona urbana da Cidade de Pelotas, Brasil. As mulheres foram selecionadas através de amostragem de multi-estágios. As questões sobre aborto induzido foram aplicadas utilizando uma das duas metodologias.

Resultados. Dentre as mulheres entrevistadas com o método da urna, 7,2\% relataram ter induzido pelo menos um aborto, enquanto que para aquelas entrevistadas com o método das questões indiretas esse valor foi de 3,8\%. A razão entre os dois métodos foi de 1,89 (IC 95\%: 1,39 a 2,60; $\mathrm{P}<0,001$ ). Ao final da vida reprodutiva (45 a 49 anos) $12 \%$ haviam induzido aborto, segundo o método da urna. Os principais motivos para as mulheres terem provocado aborto foram ter dificuldades econômicas, ser muito jovem ou ser solteira. Aproximadamente a metade das mulheres que relataram aborto através do método da urna tinha utilizado procedimentos inseguros, sendo que $13 \%$ utilizaram misoprostol.

Conclusão. O método da urna mostrou-se adequado para estudar a freqüência de aborto induzido ou outros temas que possam gerar sub-relato por parte dos entrevistados, principalmente em amostras de base populacional. É provável que a alteração na formulação da pergunta sobre aborto (por exemplo, eliminar a palavra "filho") poderia aumentar a sensibilidade do método da urna para detectar a ocorrência de aborto induzido.

Palavras-chave Aborto induzido, epidemiologia, metodologia.

Uma vez que, no Brasil, a prática de provocar aborto é amplamente condenada por preceitos éticos, morais e religiosos, a coleta de informações sobre esse procedimento geralmente não re-

\footnotetext{
1 Universidade do Vale do Rio dos Sinos (UNISINOS), Programa de Pós-Graduação em Ciências da Saúde. Correspondência para esta autora no seguinte endereço: Avenida UNISINOS 950, caixa postal 275, CEP 93022-000, São Leopoldo, RS, Brasil. E-mail: mtolinto@bios.unisinos.br

2 Universidade Estadual de Campinas, Faculdade de Ciências Médicas, Departamento de Medicina Preventiva e Social, Campinas (SP), Brasil.
}

vela a verdadeira magnitude do problema. Estudos têm demonstrado que, conforme o relato de $82 \%$ das mulheres e $84,8 \%$ dos homens, o aborto induzido só é aceito em caso de risco de vida para a mulher $(1,2)$. Portanto, inúmeras são as dificuldades no estudo da freqüência do aborto induzido. $\mathrm{O}$ subregistro pode estar presente tanto em contextos onde o aborto é ilegal quanto em situações onde essa prática é garantida pela lei (3). Da mesma maneira, as características individuais ou os aspec- tos da vida reprodutiva podem induzir a diferentes níveis de sub-relato, mesmo em países onde o aborto é permitido e pouco estigmatizado (4).

No Brasil, embora existam alguns estudos de base populacional sobre o aborto induzido $(5,6)$, a maioria deles se baseia em informações obtidas de fontes hospitalares (7-9). Uma das estimativas de aborto induzido mais citadas para o Brasil - 1,4 milhão de abortos induzidos ao ano teve como base informações de profissionais de 
saúde e registros hospitalares (10). Além disso, outros estudos foram realizados com grupos limitados de mulheres. Por exemplo, Coelho et al. (11) apresentaram características de mulheres da Cidade de Fortaleza que utilizaram misoprostol para indução do aborto, enquanto que Hardy et al. (12) descreveram características demográficas restritas a estudantes e funcionárias de uma mesma universidade no Estado de São Paulo (13).

Em 1994, na Colômbia, houve um encontro para apresentação e discussão de aspectos metodológicos na pesquisa sobre aborto induzido, especificamente para investigadores da América Latina e do Caribe. Dentre as diversas metodologias apresentadas, destacou-se o método da urna, técnica que utilizava um questionário sem identificação pessoal, garantindo total anonimato para as entrevistadas, o qual era introduzido em uma urna (14). Pouco antes disso, Huntington et al. (15) sugeriram a utilização do contexto da gravidez indesejada para abordar o aborto induzido através de questões indiretas, com a finalidade de evitar o sub-relato inerente às investigações de aborto induzido.

Assim, o objetivo do presente trabalho foi comparar uma versão adaptada do método da urna com o método de questões indiretas para estudo da freqüência de aborto induzido em uma amostra representativa de mulheres residentes no sul do Brasil.

\section{MATERIAIS E MÉTODOS}

Um estudo transversal foi realizado em 1995 com uma amostra representativa de mulheres em idade reprodutiva (15 a 49 anos) residentes na zona urbana de Pelotas, município localizado no Estado do Rio Grande do Sul. As mulheres sorteadas para participar do estudo foram entrevistadas nos seus domicílios. As informações socioeconômicas, demográficas e reprodutivas de todas as mulheres da amostra foram coletadas através de um questionário geral aplicado no início da entrevista. As informações sobre aborto induzido foram obtidas utilizando-se dois instrumentos. Através de alocação aleatória, a metade das mulheres foi selecionada para relatar sobre aborto induzido através de um instrumento denominado método da urna (MU) e a outra metade através do método das questões indiretas (MQI).

Para o cálculo do tamanho da amostra, foram realizadas várias simulações com base nas estimativas de prevalência de aborto induzido por estudos de base populacional (4 a 10\%), com um poder estatístico de 80 a $90 \%$, um erro alfa de $5 \%$ e razão de expostos para não expostos de 1:1 (devido à alocação em um entre dois instrumentos). Ao final, foi escolhido um tamanho de amostra de 3098 mulheres (1 549 em cada grupo), com uma probabilidade de $80 \%$ de detectar uma razão de prevalências de 1,5 e uma prevalência de abortos induzidos de $5 \%$, pelo método das questões indiretas. Prevendo possíveis perdas e recusas, o tamanho da amostra foi aumentado para 3200 mulheres. Para alcançar este total, sabendo-se que nessa faixa etária há cerca de 0,92 mulheres por domicílio, seria necessário visitar cerca de 3478 domicílios. Portanto, visitando 70 domicílios em 50 setores censitários (dentre os 258 existentes na cidade), seriam encontradas, em média, 64 mulheres por setor, atingindo o tamanho de amostra calculado.

A escolha dos 50 setores censitários foi feita através de uma amostragem sistemática, o que garantiria a representação das diferentes áreas da cidade e, conseqüentemente, dos diversos grupos socioeconômicos. O próximo passo no processo amostral foi a escolha do local em cada setor onde seria iniciado o trabalho de campo. Uma vez definido o ponto de partida, as entrevistadoras eram orientadas a caminhar sempre na mesma direção. Com isso procurou-se evitar um viés de seleção, isto é, entrevistar apenas as mulheres que morassem nas casas de mais fácil acesso.

Todas as entrevistadoras que trabalharam no campo eram mulheres, maiores de 18 anos, estudantes universitárias da área de ciências humanas e da saúde e não apresentavam opinião radical em relação ao aborto, seja con- tra ou a favor. No início do trabalho em cada setor, sorteava-se o método (MU ou MQI) a ser utilizado pela entrevistadora com a primeira mulher entrevistada. Para esse sorteio a entrevistadora lançava uma moeda. Se o resultado fosse "cara", a primeira mulher seria entrevistada pelo MQI; com resultado "coroa", seria utilizado o método da urna. A partir da primeira entrevista, as entrevistas subseqüentes eram realizadas utilizando alternadamente os dois métodos.

Durante o trabalho de campo, notouse que a estimativa de mulheres por domicílio havia sido superestimada. Ao invés de serem encontradas 64 mulheres de 15 a 49 anos, em 70 domicílios, foram encontradas, em média, 60 mulheres. Com isso o número total de mulheres entrevistadas foi de 3002.

\section{Método da urna}

Todas as mulheres alocadas para o $\mathrm{MU}$, após responderem o questionário geral, recebiam um pequeno pedaço de papel, que foi chamado de voto, contendo questões sobre aborto. Nesse momento, a entrevistadora explicava cada questão presente no voto e mostrava a urna na qual o voto deveria ser depositado. A urna foi desenvolvida para ser de fácil transporte durante o trabalho de campo - possui uma alça e é leve, feita de lona, com a parte superior em acrílico. Todas as urnas eram numeradas, possibilitando a identificação do entrevistador e do setor censitário associados à urna.

As entrevistadoras foram treinadas para enfatizar quatro características do método para as entrevistadas: a) não havia identificação pessoal no voto; $b$ ) como a face superior da urna era de acrílico, a entrevistada podia ver outros votos no interior da urna; c) a urna era fechada a cadeado (lacrada) e somente podia ser aberta pelos coordenadores da pesquisa; e d) seria garantida a privacidade no momento do preenchimento.

Para a garantia do item b acima, a urna continha sempre três votos em branco introduzidos pela coordenação do estudo, evitando que, no início de 
cada setor, a urna estivesse vazia e causasse constrangimento para a entrevistada. Posteriormente, esses três votos em branco eram excluídos da análise. $\mathrm{O}$ voto continha as seguintes perguntas: qual sua idade; alguma vez você tirou ou abortou um filho que você não queria ou não podia ter (sim ou não); se sim, quantos abortos você fez; em que ano foi o último aborto; quem fez o último aborto para você (médico, parteira, você mesma, outros); como foi feito.

Cada vez que uma entrevistadora concluía todas as entrevistas em um setor censitário, a urna lacrada era levada para a coordenação da pesquisa, onde seria aberta, e os votos do setor, contabilizados.

\section{Método das questões indiretas}

Esse método foi utilizado para a outra metade das mulheres, isto é, aquelas que não foram incluídas no MU. Também nesse caso as participantes responderam primeiro o questionário geral e, depois, as questões sobre aborto. O MQI inclui as seguintes questões (15): você já esteve grávida alguma vez que não podia ou não queria estar (sim, não, não sabe, sem informação); se sim, o que você fez (nada, continuou a gravidez; tentou parar a gravidez mas não conseguiu; tentou parar a gravidez e conseguiu; outra opção). Nesse caso, as respostas eram dadas oralmente à entrevistadora.

\section{Controle de qualidade dos dados e análise estatística}

O controle de qualidade dos dados incluiu os seguintes passos: a) toda a codificação de questionário era feita no mesmo dia da entrevista, permitindo que a entrevistadora fosse capaz de lembrar da entrevista e identificar e corrigir qualquer engano. Se houvesse necessidade, a entrevistadora poderia ainda repetir a entrevista no dia seguinte; b) a cada 3 dias, as entrevistadoras entregavam os questionários para os revisores, que conferiam todas as respostas e a codificação. Em caso de erro ou inconsistência era requerida a volta ao domicílio; c) o supervisor de trabalho de campo refez 5\% das entrevistas. Além da veracidade das informações, era conferida também a planilha de trabalho de campo; e d) todos os questionários foram digitados duas vezes, por digitadores diferentes, para evitar erros.

Os dados foram digitados utilizandose o programa Epi Info (16). A inconsistência dos dados foi verificada utilizando-se a função check do mesmo programa. Para serem analisados, os dados foram transferidos para o Statistical Package for the Social Sciences (SPSS) (17). A análise estatística compreendeu os seguintes passos: a) comparação das características socioeconômicas, demográficas, reprodutivas e relativas a aborto induzido entre os dois grupos de mulheres alocadas para o MU ou MQI; b) comparação da estimativa de aborto induzido obtida com cada um dos métodos; c) descrição dos procedimentos utilizados para o aborto induzido e d) descrição dos motivos para a realização do aborto.

\section{RESULTADOS}

A partir dos 50 setores censitários sorteados para o estudo, 3857 domicílios foram visitados e 3154 mulheres de 15 a 49 anos foram localizadas. Após as recusas ou perdas (impossi- bilidade de contatar depois de três tentativas) foram incluídas no estudo 3002 mulheres. Dessas, 1492 mulheres foram entrevistadas com o MU e 1510 com o MQI. A diferença entre o número de mulheres entrevistadas com cada um dos dois métodos foi conseqüência do procedimento de alocação.

Os dois grupos foram semelhantes quanto às características socioeconômicas, demográficas e reprodutivas - não foram encontradas diferenças estatisticamente significativas, fato que indica sucesso na alocação aleatória. Os dois grupos tinham em média 31 anos de idade e 8,5 anos de escolaridade; $84 \%$ das mulheres eram brancas; cerca de $56 \%$ eram casadas ou viviam em união; $30 \%$ viviam com menos de três salários mínimos de renda familiar (sendo o salário mínimo equivalente a 100 dólares na época); e cerca de $50 \%$ trabalhavam. Em ambos grupos, $86 \%$ tinham vida sexual ativa e 1,4 filho em média.

De acordo com o MU, 107 (7,2\%) mulheres relataram aborto induzido, enquanto que com o MQI esse valor foi de $58(3,8 \%)$ mulheres. A razão entre os dois métodos foi de 1,89 (IC 95\%: 1,39 a 2,60; $P<0,001$ ). Esses valores foram semelhantes aos resultados preliminares divulgados previamente como nota de pesquisa pela investigadora principal (MTAO) (18).

A figura 1 compara o relato de aborto induzido entre os dois métodos

\section{FIGURA 1. Comparação do relato de aborto induzido através do método da urna e do método das questões indiretas de acordo com a faixa etária, Pelotas (RS), Brasil, 1995a}

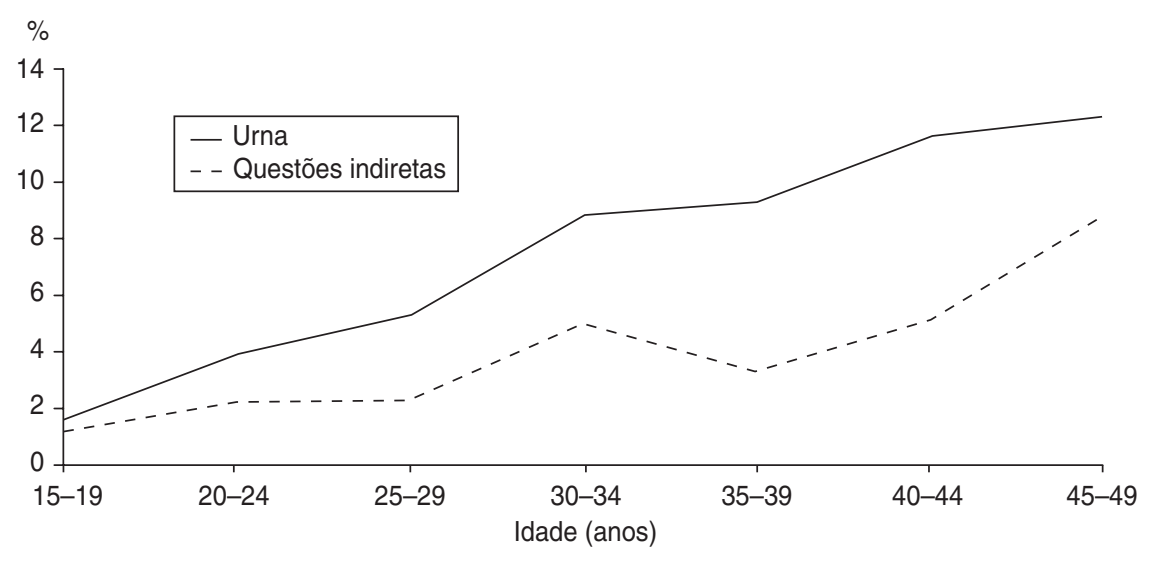

a Foram entrevistadas 3002 mulheres. 
para cada faixa etária. Para todas as faixas etárias, o MU produziu maiores estimativas, sendo que, para a faixa etária de 45 a 49 anos, 12,3\% das mulheres entrevistadas por esse método relataram ter induzido pelo menos um aborto. Uma achado interessante foi a maior diferença entre as taxas obtidas pelos dois métodos para a faixa etária de 35 a 44 anos, atingindo uma razão de 2,8 .

De acordo com o MQI, os motivos mais freqüentes para indução do aborto foram questões econômicas $(31 \%)$ e ser muito jovem ou solteira (24\%). Dentre as 107 mulheres que relataram aborto induzido pelo MU, o método mais utilizado foi curetagem ou aspiração. A droga misoprostol (Cytotec) foi empregada em 13\% dos abortos.

\section{DISCUSSÃO}

Criatividade e combinação de metodologias têm sido apontadas como caminhos para o estudo do aborto induzido em países com leis restritivas (19). Conforme utilizado no presente estudo, o MU, embora semelhante à proposta de Zamudio et al. (14), apresenta um aspecto inovador: a utilização de acrílico na parte superior da urna, permitindo a visualização de outros votos. Essa particularidade é importante principalmente quando se utilizam entrevistas domiciliares. Em relação ao MQI, nosso estudo mostrou a pouca efetividade desse método para estimar aborto induzido, principalmente em estudos de base populacional. O artigo de Huntington et al. (20), onde é descrita a aplicação deste método em mais de um país, também evidenciou a fragilidade do mesmo.

No presente estudo, como as mulheres alocadas para cada um dos métodos não apresentaram diferenças em termos socioeconômicos, reprodutivos e demográficos, pode-se inferir que a diferença entre as estimativas de aborto - o número de abortos relatados a partir do MU foi o dobro do número de abortos relatado pelo MQI - é atribuível ao próprio instrumento. Mesmo assim, acreditamos que mesmo a estimativa do MU esteja subestimada, e algumas considerações devem ser feitas.

Considerando todas as faixas etárias, foi constatado, pelo $\mathrm{MU}$, que 7,2\% das mulheres haviam feito pelo menos um aborto durante sua vida reprodutiva. Entretanto, deve-se levar em conta que a maioria das mulheres na amostra ainda não havia completado sua vida reprodutiva; levando em conta apenas o grupo de 45 a 49 anos, o estudo mostrou que $12 \%$ das mulheres tinham induzido pelo menos um aborto. Mesmo assim, a estimativa da freqüência de aborto induzido foi menor do que aquela relatada anteriormente pelo Allan Guttmacher Institute (AGI) para o Brasil como um todo, de 1,4 milhão de abortos induzidos por ano (10). Isso poderia ser explicado pelas diferenças metodológicas entre esses dois estudos: enquanto o presente trabalho foi baseado em dados populacionais, o estudo do AGI partiu de dados obtidos junto a serviços e profissionais de saúde. Por outro lado, mesmo que a estimativa obtida pelo MU possa estar subestimada, deve-se levar em consideração os achados do estudo de Corrêa e Freitas (21), que revelam uma queda no número de abortamentos induzidos para o Estado do Rio Grande do Sul de 28 e $39 \%$, respectivamente, para os períodos de 1994 a 1995 e 1995 a 1996. Corrêa e Freitas empregaram dados do sistema de informações DATASUS, do Ministério da Saúde do Brasil, sobre procedimentos de curetagem pós-abortamento e, posteriormente, aplicaram aos dados os mesmos cálculos adotados pelo AGI.

Dois outros estudos brasileiros de base populacional também mostraram estimativas superiores às obtidas no presente estudo com o MU. O primeiro foi um estudo etnográfico realizado por Leal e Fachel (22) com repetidas entrevistas, mostrando que $18 \%$ das mulheres tinham induzido pelo menos um aborto; entretanto, essa metodologia consome maior tempo e não pode ser aplicada em amostras grandes. $\mathrm{O}$ segundo estudo (23) utilizou a técnica de resposta ao azar (TRA) e mostrou uma incidência anual de 4,1\% entre as mulheres em idade reprodutiva em São Paulo, e 31,3\% das gestações terminando em aborto. Contudo, a aplicação desta técnica pode ser considerada bem mais complexa do que a utilização de uma urna.

Outros estudos de base populacional, utilizando questionários, têm mostrado taxas consideravelmente baixas. Por exemplo, um estudo realizado em Campinas, Estado de São Paulo (6), estimou que $4 \%$ das mulheres haviam provocado um aborto. Nesse caso, a análise foi limitada às mulheres que alguma vez estiveram grávidas. Cesar e Horta (24), que avaliaram uma amostra representativa de 1456 mulheres de 15 a 49 anos em uma cidade próxima a Pelotas, encontraram um resultado semelhante ao apresentado aqui, com prevalência de $20 \%$ de abortamento durante a vida, sendo que um terço deles seriam provocados.

$\mathrm{O}$ estudo de Costa et al. (25) trabalhou com uma amostra de alunas e funcionárias de uma universidade no Brasil. Utilizando um questionário auto-aplicado sem identificação pessoal, esse estudo teve apenas 30\% de devolução dos questionários enviados, sendo maior o percentual de devolução entre as alunas. Desconsiderando o viés de não-resposta (viés de seleção), a estimativa de aborto induzido encontrada no estudo foi de cerca de $11 \%$ entre o total de respondentes (2 924 mulheres). Supondo que as mulheres não respondentes desse questionário fossem aquelas que, numa entrevista direta, iriam informar que não realizaram aborto, teríamos uma estimativa de aborto induzido de 3,6\% (347/9 590), valor próximo aos 3,8\% observado por nós com o método das questões indiretas.

$\mathrm{Na}$ comparação das estimativas de abortamento, existem basicamente dois tipos de problemas: as diferentes medidas utilizadas pelos pesquisadores (tais como prevalência durante a vida e incidência anual) e as amostras diferenciadas. Na comparação dos resultados, deve-se levar em conta que os estudos foram realizados em diferentes partes do país, o que pode implicar diferentes taxas, por exemplo: áreas metropolitanas podem ter taxas 
muito mais elevadas do que uma cidade de porte médio, tal como a Cidade de Pelotas.

Outro fator limitante da MU foi a forma como foi elaborada a pergunta sobre aborto. Conforme mencionado anteriormente, a pergunta sobre aborto induzido no MU foi: "Alguma vez você tirou ou abortou um filho que não queria ou não podia ter tido?" É possível que a palavra "filho" tenha reduzido a chance de relato, ocasionando um grande número de falsos negativos e levando a uma baixa estimativa do aborto induzido. Essa possibilidade pode ser amparada pelo estudo de Leal e Lewgoy (26), no qual foi salientada a existência de diferenças no entendimento por parte das mulheres do que seria ou não considerado uma gravidez e, conseqüentemente, um aborto.

Aproximadamente a metade das mulheres que relataram aborto através do MU tinham utilizado procedimentos inseguros, sendo que $13 \%$ utilizaram misoprostol. A escolha desta droga pelas mulheres deve-se basicamente ao seu baixo custo em comparação a outros métodos e à possibilidade da realização do aborto com privacidade, geralmente com pouca interferência externa (27). Os métodos utilizados para o aborto diferem um pouco em relação ao estudo de Cesar e Horta (24). Naquele estudo, em $25 \%$ dos abortos foi utilizado misoprostol, em $20 \%$ sondas e em $13 \%$ dos abortos foi utilizada a curetagem. Neste estudo, as estimativas foram de 13, 6 e 52\% para cada um desses métodos, respectivamente. $\mathrm{O}$ menor percentual do uso de misoprostol pode ser atribuído à melhor escolaridade e renda das mulheres em nosso estudo, levando-as a uma preferência levemente mais elevada pelos métodos de aborto com menor risco. Outra provável explicação seria o fato de essa informação ter sido obtida através de autoresposta - as mulheres que iniciaram o aborto com o misoprostol e, posteriormente, tiveram que recorrer a um hospital, onde foi realizada uma curetagem a fim de concluir o procedimento, informaram no "voto" apenas o dado de curetagem. Um estudo realizado em Fortaleza encontrou que $58 \%$ dos abortos realizados com misoprostol recorrem posteriormente a curetagem (11).

Os principais motivos para as mulheres terem provocado aborto foram ter dificuldades econômicas e ser muito jovem ou solteira. Como era esperado, induzir o aborto por motivos econômicos foi mais freqüente neste estudo do que naquele com alunas e funcionárias de uma universidade em
São Paulo (25), mas observou-se similar proporção de mulheres que atribuíram o aborto ao fato de serem solteiras. $\mathrm{O}$ estudo realizado na universidade apresentou como principal motivo o fato de "não estar preparada para criar / educar," o que, em última instância, implica incompatibilidade do ser mãe com projetos pessoais, uma resposta considerada adequada para mulheres inseridas em uma universidade.

Na comparação entre o MU e o MQI para estimar a freqüência de aborto induzido, embora possivelmente tenha havido sub-relato com ambas as técnicas, a urna de acrílico mostrou-se como uma solução razoável para pesquisas de base populacional sobre temas que envolvem questões éticas, morais e religiosas. Além disso, a sensibilidade desta metodologia, isto é, a capacidade do método da urna de detectar o aborto induzido em situações em que realmente ele ocorreu, poderia ser aumentada modificando-se a redação da pergunta sobre indução de aborto.

Agradecimentos. Os autores são gratos ao Population Council (escritório de Nova Iorque) e ao Conselho Nacional de Desenvolvimento Científico e Tecnológico $(\mathrm{CNPq})$ pelo apoio financeiro (processo 142783/96-0).

\section{REFERÊNCIAS}

1. Vieira EM. Do women's attitudes towards abortion and contraceptive methods influence their option for sterilization? Cad Saude Publica. 1999;15(4):739-47.

2. Duarte GA, Alvarenga AT, Osis MJ, Faúndes A, Hardy E. Perspectiva masculina acerca do aborto provocado. Rev Saude Publica. 2002; 36(3):271-7.

3. Jones EF, Forrest JD. Underreporting of abortion in surveys of US women: 1976 to 1988. Demography. 1992;29(1):113-26.

4. Anderson B, Katus K, Puur A, Silver BD. The validity of survey responses on abortion: evidence from Estonia. Demography. 1994;31(1): $115-32$.

5. Silva RS. Incidencia y características del aborto inducido en São Paulo. Em: Encuentro de Investigadores sobre Abortos Inducidos en América Latina y Caribe. Aspectos metodológicos. Bogotá: Universidad Externado de Colombia; 1994.

6. Osis MJD, Hardy E, Faúndes A, Rodrigues T. Dificuldades para obter informações da popu- lação de mulheres sobre aborto ilegal. Rev Saude Publica. 1996;30(5):444-51.

7. Fonseca W, Misago C, Correia LL, Parente JAM, Oliveira FC. Determinantes de aborto induzido entre mulheres admitidas em hospitais em localidade da região Nordeste do Brasil. Rev Saude Publica. 1996;30(1): 13-8.

8. Singh S, Wulf D. The likelihood of induced abortion among women hospitalized for abortion complications in four Latin American countries. Int Fam Plann Perspect. 1993;19(4): $134-41$.

9. Schor N. Investigação sobre a ocorrência de aborto em pacientes de hospital de centro urbano do Estado de São Paulo, Brasil. Rev Saude Publica. 1990;24(2):144-51.

10. Alan Guttmacher Institute (AGI). Aborto clandestino: uma realidade latino-americana. Nova Iorque: The Alan Guttmacher Institute; 1994.

11. Coelho HL, Teixeira AC, Cruz MD, Gonzaga SL, Arrais PS, Luchini L, et al. Misoprostol: the experience of women in Fortaleza, Brazil. Contraception. 1994;49(2):101-10.

12. Hardy E, Costa RG, Rodrigues T, Moraes TM Características atuais associadas à história de aborto provocado. Rev Saude Publica. 1994; 28(1):82-5.

13. Hardy E, Rebello I, Faúndes A. Aborto entre as alunas e funcionárias de uma universidade brasileira. Rev Saude Publica. 1993;27(2): 113-6.

14. Zamudio L, Rubiano N, Wartenberg L. Representatividad, confiabilidad y significación: problemas prácticos de la investigación sobre aborto inducido. Em: Encuentro de Investigadores sobre Abortos Inducidos en América Latina y Caribe. Determinantes del aborto y factores asociados. Santa Fe de Bogotá: Universidad Externado de Colombia; 1994.

15. Huntington D, Mensch B, Toubia N. A new approach to eliciting information about induced abortion. Stud Fam Plann. 1993;24(2): 120-124.

16. Dean JG, Dean JA, Coulombier D, Brendel KA, Smith DC, Burton AH, et al. Epi Info 6.0: 
a word processing database and statistics program for epidemiology on IBM compatible computers. Atlanta, Georgia: Centers for Disease Control and Prevention; 1994.

17. SPSS. SPSS for Windows - release 8.0.0. Chicago: SPSS Inc; 1988.

18. Olinto MTA. Estimativa da freqüência de abortos induzidos: teste de uma metodologia. Rev Bras Estudos Pop. 1994;11(2):255-8.

19. Coeytaux F. Methodological issues in abortion research. Nova Iorque: The Population Council; 1989.

20. Huntington D, Mensch B, Miller V. Survey questions for the measurement of induced abortion. Stud Fam Plann. 1996;27(3):155-61.

21. Corrêa S, Freitas A. Atualizando os dados sobre a interrupção voluntária da gravidez no Brasil. Estudos Feministas. 1997;5(2):389-95.
22. Leal O, Fachel J. Corpo, sexualidade e reprodução: um estudo de representações sociais em quatro vilas de Porto Alegre/RS Brasil. Em: World Health Organization Final Report to Special Programme of Research, Development and Research Training in Human Reproduction. Genebra: WHO; 1995.

23. Silva RS. O uso da Técnica de Resposta ao Azar (TRA) na caracterização do aborto ilegal. Rev Bras Estudos Pop. 1993;10(1/2):41-56.

24. Cesar J, Horta BL. Desigualdade e perversidade: epidemiologia do adoecer no extremo sul do Brasil. Rio Grande: Editora da FURG: 1997.

25. Costa RG, Hardy E, Osis MJD, Faúndes A. A decisão de abortar: processo e sentimentos envolvidos. Cad Saude Publica. 1995;11(1):97105.
26. Leal O, Lewgoy B. Aborto: uma contribuição antropológica à discussão. Em: Filosofia política: nova série. Vol 2. Porto Alegre: L\&PM; 1998. Pp. 173-95.

27. Barbosa RM, Arrilha M. The Brazilian experience with Cytotec. Stud Fam Plann. 1993; 24(4):236-40.

Manuscrito recebido em 3 de setembro de 2003. Aceito em versão revisada em 28 de janeiro de 2004.

ABSTRACT Objective. To compare two methods-the "ballot box" method and the "indirect questioning" method-for estimating the frequency of induced abortions in population-based studies.

Estimating the frequency of induced abortion: a comparison of two methods
Methods. A cross-sectional population-based study was conducted with a representative sample of 3002 women between 15 and 49 years of age living in the city of Pelotas, Rio Grande do Sul, Brazil. The women were selected through multistage sampling and randomly assigned to answer questions concerning induced abortion with one of the two methods, after they had answered a general questionnaire that collected socioeconomic and demographic information. With the "ballot box" method, women received a small piece of paper containing direct questions on abortion. Each woman marked her answers on the paper and then deposited it into a small "ballot box" carried by the interviewer, thus assuring the confidentiality of the responses. With the second method, the interviewer verbally asked the woman a series of questions that indirectly inquired about abortion.

Results. Among the women assigned to the ballot box method, 7.2\% reported having induced at least one abortion, versus $3.8 \%$ of the women assigned to the indirect questioning method. The ratio between the two methods was 1.89 ( $95 \%$ confidence interval: 1.39 to $2.60 ; P<0.001$ ). Of the women surveyed who were $45-49$ years old (at the end of their reproductive life) and answered using the ballot box method, $12 \%$ of them reported having had at least one induced abortion in their lifetime. Among the women who answered using the ballot box method, the main reasons they gave for having had an abortion were economic difficulties, being too young, and being single. Approximately half of the women responding to the ballot box method questions reported they had used unsafe procedures to induce abortion, with $13 \%$ of them using misoprostol.

Conclusions. The ballot box method was suitable for studying the frequency of induced abortion and for researching other topics that may lead to underreporting by the persons interviewed, especially in population-based samples. Rephrasing the ballot box method question about abortion (for example, eliminating the word "child") would probably increase the method's sensitivity, that is, its ability to detect induced abortions that had occurred. 